\title{
A utilização dos massageadores perineais e dilatadores vaginais como métodos de tratamento fisioterapêutico nas Disfunções Pélvicas: Vaginismo e Dispareunia
}

The use of perineal massagers and vaginal dilators as methods of physiotherapeutic treatment in

\author{
Pelvic Dysfunctions: Vaginismus and Dyspareunia
}

El uso de masajeadores perineales y dilatadores vaginales como métodos de tratamiento

fisioterapéutico en las disfunciones pélvicas: vaginismo y dispareunia

Recebido: 12/05/2021 | Revisado: 17/05/2021 | Aceito: 18/05/2021 | Publicado: 04/06/2021

\author{
Bruna Pereira Nagamine \\ ORCID: https://orcid.org/0000-0002-8799-2529 \\ Faculdade Guaraí, Brasil. \\ E-mail: brunapnagamine@gmail.com \\ Karla Camila Correia da Silva \\ ORCID: https://orcid.org/0000-0003-1538-7028 \\ Faculdade Guaraí, Brasil. \\ E-mail: karlacamilac@yahoo.com.br
}

\begin{abstract}
Resumo
Os massageadores perineais são recursos manuais terapêuticos que são realizados através de deslizamentos e liberações miofasciais na região pélvica, estimulando o relaxamento da musculatura e provocando um efeito inibitório nas tensões musculares, e os dilatadores vaginais amolecem os tecidos e promovem consciência corporal. O objetivo do presente artigo é demonstrar como os dos massageadores perineais e dilatadores vaginais podem ser eficazes no tratamento do vaginismo e da dispareunia, a partir das pesquisas realizadas através das plataformas digitais: Scielo, Pubmed e PEDro. Conclui-se que diante das patologias vaginismo e dispareunia os massageadores perineais e dilatadores vaginais podem ser eficazes no tratamento por diminuindo a percepção da dor e a tensão muscular, recuperando o tônus e promovendo a dessensibilização da musculatura do assoalho pélvico.
\end{abstract}

Palavras-chave: Fisioterapia; Saúde da mulher; Assoalho pélvico.

\begin{abstract}
Perineal massagers are manual therapeutic resources that are carried out through slips and myofascial releases in the pelvic region, stimulating the relaxation of the muscles and causing an inhibitory effect on muscular tensions, and the vaginal dilators soften the tissues and promote body awareness. The purpose of this article is to demonstrate how those of perineal massagers and vaginal dilators can be effective in the treatment of vaginismus and dyspareunia, based on research carried out through the digital platforms: Scielo, Pubmed and PEDro. It is concluded that in the face of vaginismus and dyspareunia pathologies, perineal massagers and vaginal dilators can be effective in the treatment by decreasing the perception of pain and muscle tension, recovering tone and promoting the desensitization of the pelvic floor muscles.
\end{abstract}

Keywords: Physiotherapy; Women's health; Pelvic floor.

\section{Resumen}

Los masajeadores perineales son recursos terapéuticos manuales que se realizan a través de deslizamientos y liberaciones miofasciales en la región pélvica, estimulando la relajación de los músculos y provocando un efecto inhibitorio sobre las tensiones musculares, y los dilatadores vaginales suavizan los tejidos y promueven la conciencia corporal. El propósito de este artículo es demostrar cómo los masajeadores perineales y dilatadores vaginales pueden ser efectivos en el tratamiento del vaginismo y la dispareunia, a partir de investigaciones realizadas a través de las plataformas digitales: Scielo, Pubmed y PEDro. Se concluye que ante patologías de vaginismo y dispareunia, los masajeadores perineales y dilatadores vaginales pueden ser efectivos en el tratamiento disminuyendo la percepción de dolor y tensión muscular, recuperando el tono y favoreciendo la desensibilización de la musculatura del suelo pélvico. Palabras clave: Fisioterapia; La salud de la mujer; Piso pelvico. 


\section{Introdução}

O assoalho pélvico proporciona sustentação a dos órgãos pélvicos, como também participa no mecanismo de fechamento e abertura da uretra, vagina e ânus (Mannella et al, 2013). A sustentação dá-se, em grande parte, pelas relações complexas e dinâmicas dos músculos e do tecido conjuntivo dentro da região interna da pelve (Maldonado \& Wai, 2016).

O vaginismo é a contração involuntária da musculatura do assoalho pélvico, especificamente os músculos externos da vagina, fazendo com que dificulte ou impeça a penetração gerando dor provocado pela contração muscular involuntária e devido a isso as mulheres perdem o desejo sexual e apresentam déficits nas respostas sexuais. (Palma, et. al. 2014).

A dispareunia é definida por dor genital associada à relação sexual, no entanto, pode acontecer antes ou após à relação podendo ser provocada por alterações fisiológicas ou psicológicas sendo considerada disfunção sexual prejudicando a satisfação sexual das mulheres (Mendonça et al 2012). Esta disfunção apresenta sintomas como: queixa de dor persistente, recorrente ou desconforto associado com tentativa ou a completa penetração vaginal (Haylen, 2010).

Suas origens estão relacionadas a causas psicológicas, geniturinárias, neurológicas, traumas, abuso sexual, transtornos emocionais, falta de conhecimento e diálogo com o parceiro. É também pode ser levado em consideração os fatores etiológicos: drogas, sedativos, tranquilizantes, hormonais (Etienne; Waitman, 2006; Antonioli; Simões, 2010).

Segundo a Organização Mundial de Saúde (2012), quando trata de disfunções sexuais as mesmas interferem de forma direta na vida social das mulheres, devido a isso é considerada uma problemática na saúde pública. Quando a mulher tem um déficit nas suas respostas sexuais é denominado disfunção sexual, seja ela na fase do desejo, lubrificação, orgasmo, excitação e dor na tentativa ou durante $o$ ato sexual.

No Brasil assim como em diversos outros países, ainda existe predominância de uma forte cultura repressora se tratando de saúde sexual tanto no âmbito familiar, quanto no social e religioso, o que impossibilita ainda mais a transmissão do conhecimento a respeito do vaginismo e dispareunia assim como o avanço dos estudos, diagnósticos e possíveis tratamentos terapêuticos satisfatório (Goulart, 2013).

A terapia manual é constituída por um conjunto de técnica com intenção terapêutica, com o objetivo de desfazer trigger points, promover o relaxamento, aumentar a coordenação e propriocepção da musculatura e aumentar o fluxo sanguíneo local (Prendergast; 2008). E a utilização dos dilatadores vaginais como recurso de dilatação gradual como princípio auxiliar na dessensibilizarão do canal vaginal, reduzir a tensão muscular, melhorando o conforto durante a relação sexual (Lucheti et al, 2019).

Dessa forma, a fisioterapia pélvica é fundamental na prevenção e tratamento das disfunções pélvicas, melhorando os sintomas em pacientes com vaginismo e dispareunia, pois é, eficiente, promovendo a redução dos espasmos musculares, melhorando a consciência e conhecimento corporal influenciando na diminuição das contrações involuntárias, aliviando a dor no ato sexual (Tomen; et al, 2016).

\section{Metodologia}

Este estudo trata-se de uma revisão bibliográfica, de característica descritiva e exploratória, com abordagens qualitativas. Tendo como princípio para sua discussão teórica, artigos científicos (Pereira et al., 2018). O levantamento de dados foi realizado através das bases de dados: Scielo, Pubmed e PEDro, no período de fevereiro de 2021 a março de 2021. Foram utilizadas as palavras chaves em português e inglês: disfunções sexuais, assoalho pélvico, fisioterapia pélvica. Foram encontrados 58 artigos, e selecionados apenas 27 foram para a pesquisa e os textos examinados foram criteriosamente avaliados por dois autores independentes.

Foram considerados os critérios de inclusão: artigos e produções intelectuais que demonstrasse eficiência como forma de tratamento de acordo com a sintomatologia que pacientes com vaginismo e dispareunia apresentam. 


\section{Discussão teórica}

\section{Assoalho Pélvico}

A pelve é constituída pelo cóccix, sacro e dois ossos ilíacos e toda essa estrutura óssea formam duas cavidades: a superior e a inferior. A cavidade superior é mais rasa e maior, nomeada pelve falsa, juntamente com órgãos abdominais. E a cavidade inferior é mais profunda e menor, denominada pelve verdadeira, envolve o sistema genital, sistema genital, parte dos ureteres, bexiga e a porção final do tubo digestório. A pelve proporciona proteção aos órgãos pélvicos, distribuindo o peso para os membros inferiores, para o acetábulo durante a posição ereta (Baracho, Elza 2012).

A parte externa da genitália feminina é constituída pelo monte pubiano, grandes e pequenos lábios, vestíbulo e clitóris. E os órgãos constituído pelo sistema reprodutor feminino são: tubas uterinas, ovários, útero e vagina. A pelve tem a sua estabilidade gerada pelos ligamentos, articulações e músculos. Os músculos mais profundos são os pubococcígeo anterior, puborretal e ileococcíneo posterior que compõe os músculos levantadores do ânus, que tem como principal função estruturar e fornecer suporte durante a ação esfincteriana do canal vaginal e anal, fornecendo o aumento do fluxo sanguíneo local através dos ramos da artéria pudenda interna com inervação dos ramos do terceiro e quarto nervo sacros e nervo pudendo. Os músculos isquiocavernoso, esfincteres anais, transverso superficial do períneo e bulbocavernoso, são os músculos superficiais urogenitais (Baracho, Elza 2007).

Para permanecer a posição apropriada da pelve é importante a interação dos músculos flexores e extensores de tronco associado com as do quadril (Baracho, Elza 2012).

\section{Fisiopatologia da Doença}

\section{Vaginismo}

Quando há uma contração involuntária dos músculos da vagina conceitua-se como vaginismo, acontece em intervalos regulares ou constante, indispondo qualquer tipo de penetração vaginal, seja por ato sexual, exame ginecológico, dedo e/ou objeto. Essa contração constante não exige obrigatoriamente a mulher com vaginismo perder o desejo sexual, porém muitas delas podem apresentar algum déficit nas respostas sexuais, como por exemplo a falta do orgasmo e excitação (Rao; Nagaraj, 2015).

Dependendo da intensidade da contração que a mulher apresentar, o vaginismo pode ser identificado como leve, onde a penetração é até possível, no entanto de maneira dolorosa e difícil; moderado, quando o nível de intensidade da contração proporciona uma penetração parcial e de grave intensidade, onde a penetração vaginal é totalmente impedida. Também, é muito utilizada a divisão em vaginismo primário e secundário (Perfetti; Nardi; Arimatea, 2014).

$O$ vaginismo pode ser classificado em:

Primário: quando ainda não houve nenhum tipo de penetração, e está relacionada com a virgindade;

Secundaria: ocorre quando a mulher que anteriormente conseguia ter a penetração e devido algum trauma, infecção, pós parto ou causas emocionais/psicológicas não consegue mais ou consegue porem, com muita dor (Aveiro; et. al. 2009).

\section{Dispareunia}

A dispareunia por se tratar de dor genital persistente que ocorre durante ou após o ato sexual tem como prováveis causas dessa disfunção estão associadas as alterações na região pélvica durante o parto, endometriose, doenças inflamatórias pélvicas e vaginais, além de infecções nessas regiões, problemas associados a fatores psicológicos como depressão e abuso sexual e a ocorrência de vulvodínia (Lima et al, 2016). O termo dispareunia é usado para caracterizar a dor durante o estímulo sexual, podendo ser classificada em primária e secundária, onde a primária é definida como dor durante a tentativa do ato sexual, ocorrendo exclusivamente devido à incompatibilidade entre o tamanho do pênis ou o que penetra e o tamanho da vagina, em condições superficiais e profundas, também está relacionada a fatores psicológicos. A secundária é ocasionada por alterações 
fisiológicas, provocando dor ao toque e ou à movimentação e a qualquer tipo de penetração, sendo insuficiente para gerar uma distensão vaginal, mesmo que seja na primeira relação sexual. (Aquino, 2019).

No entanto, uma mulher pode ter dispareunia primária e secundária ao mesmo tempo, se tornando mista apresentando qualquer disfunção pélvica a fatores psicológicos ou alterações fisiológicas causando dor ao toque, a movimentação e durante a penetração (Matthes, 2019).

\section{Tratamento fisioterapêutico}

O tratamento fisioterapêutico é de suma importância, sendo realizado com base na sintomatologia da disfunção e prevenindo futuros agravos, embora cada paciente seja único mesmo que possuam a mesma disfunção, o fisioterapeuta tem a obrigação de traçar os objetivos de acordo com a individualidade dos pacientes e realizar condutas adequadas respeitando as particularidades de cada uma delas de acordo com os feedbacks da paciente.

Os recursos selecionados para o estudo foram os massageadores perineais sendo um método fisioterapêutico manual realizado através de deslizamentos e liberações miofasciais de nódulos de tensão, na região pélvica, provocando um efeito inibitório nas tensões musculares, produzindo o relaxamento da musculatura envolvida e o alongamento de forma progressiva, melhorando o grau de algia, consequentemente reduzindo a resistência muscular favorecendo a penetração (Lucheti et al, 2019). O método deve ser realizado em contato com a pele e regiões em volta da vulva mais com ênfase maior perto do canal vaginal, para proporcionar o relaxamento progressivo da musculatura pélvica e dos tecidos adjacentes (Silva e Abreu, 2014).

A massagem se mostra eficiente por favorecer a normalização do tônus muscular por meio de ações reflexas e mecânicas, aumentando a circulação sanguínea local, melhorando a flexibilidade muscular e o fluxo linfático (Delgado et al, 2015).

Os dilatadores vaginais é um método de dilatação gradual, onde irão ser introduzidos dilatadores fabricados de silicone e para o uso deve ser utilizado sempre com camisinha e lubrificados com lubrificante para que possa ser introduzido no canal vaginal e a princípio, no início do tratamento se inicia pelos dilatadores menores e conforme a evolução da paciente vai se aumentando gradativamente, além dos dilatadores os dedos podem ser utilizados como método dilatador (Tomen et al, 2015).

A sequência de exercícios se inicia no momento da introdução do dilatador e sustentar o mesmo sem movimentar dentro do canal vaginal por 5 a 15 minutos, conforme a tolerância da paciente durante a primeira sessão terapêutica, na segunda semana o exercício pode começar a avançar, introduzindo e retirando parcialmente, em seguida, na terceira semana, a introdução e retirada do dilatador pode ser completa mediante o limiar de dor, podendo ser necessário voltar à etapas anteriores ou demorar um período maior em alguma das etapas, caso a paciente sinta dificuldade e/ou dor (Araujo e Scalco, 2019).

Os dilatadores vaginais associados com métodos de relaxamento muscular para pacientes que apresentam disfunções sexuais como o vaginismo, tem como proposito de promover o alongamento do introito vaginal além de, diminuir ou prevenir ansiedade no ato da penetração (Silva \& Abreu, 2014). 
Figura 1 - Massageador Perineal.

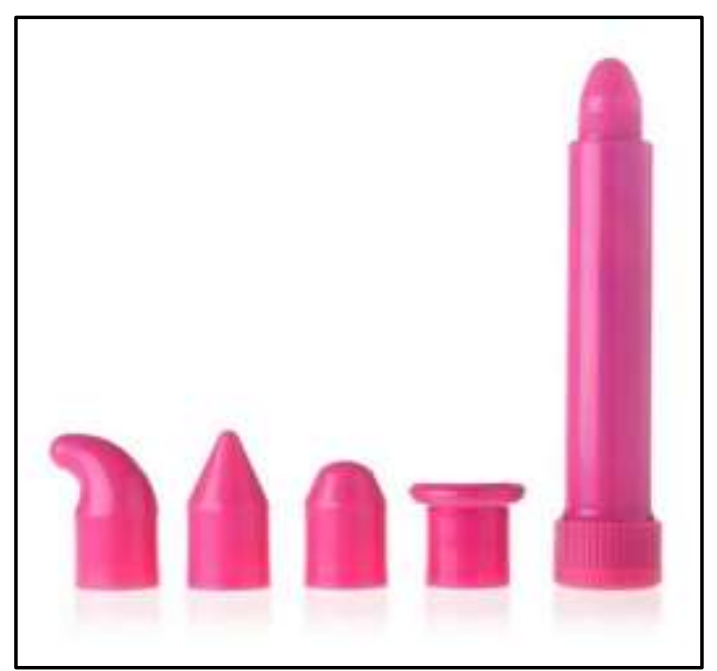

Fonte: https://www.smafisioterapia.com.br/produto/fisioterapia/uroginecologia/peridell-massageador-terapeutico-de-perineo-hot-flowers/275/

Figura 2 - Dilatadores Vaginais.

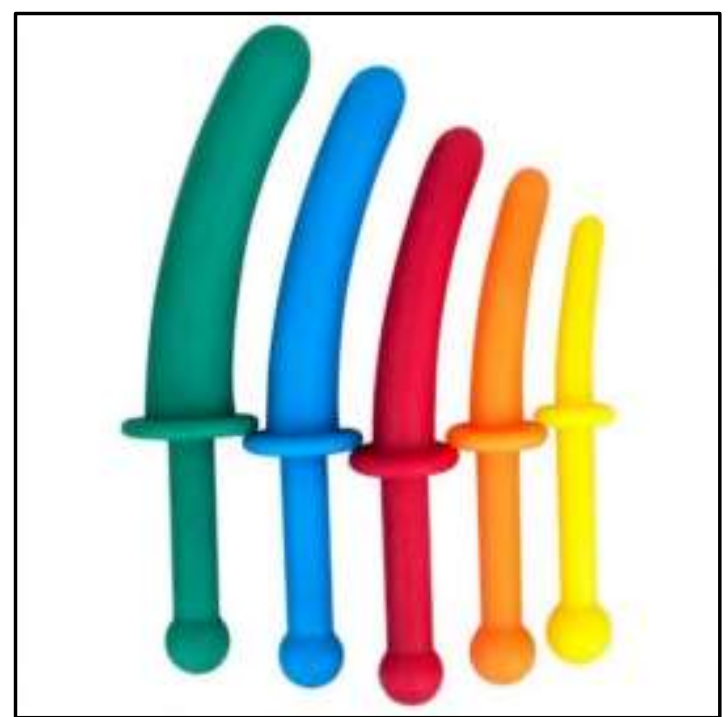

Fonte: https://www.smafisioterapia.com.br/produto/fisioterapia/uroginecologia/dell-dilatadores-vaginais-e-anais-hot-flowers/309/

\section{Conclusão}

A fisioterapia pélvica é uma área recente e pouco estudada, devido a isso, são poucos os estudos que abordam o tratamento das disfunções sexuais com ênfase no vaginismo e na dispareunia. Através do tratamento fisioterapêutico utilizando dos massageadores perineais e dilatadores vaginais essas pacientes vão melhorar o fluxo sanguíneo da região perineal, a percepção da musculatura do assoalho pélvico, permitindo melhorar o controle da musculatura e a percepção, promover o relaxamento e a educação sexual, desfazendo nódulos de tensão, dessensibilizar a região perineal e o canal vaginal, diminuir a percepção da dor e a tensão muscular, alongar e fortalecer a musculatura, além de, recuperar o tônus normal da musculatura.

O tema abordado possui relevância cientifica por se tratar de um assunto que ainda não é abordado na rede cientifica e que tem muita importância por proporcionar satisfação sexual e qualidade de vida das mulheres com vaginismo e dispareunia.

\section{Referências}

Aquino, L. H. D. C. (2019) Intervenções fisioterapêuticas na dispareunia. Faculdade de Educação e Meio Ambiente, Ariquemes-RO, 2019. 
Antonioli, R. S., \& Simões, D. (2010) Abordagem fisioterapêutica nas disfunções sexuais femininas. Rev Neurocienc. 18(2), $267-274$.

Araujo, T. G. D.; \& Scalco, S. C. P. (2019). Transtornos de dor gênito-pélvica/penetração: uma experiência de abordagem interdisciplinar em serviço público. Revista Brasileira de Sexualidade Humana, 30(1), 54-65.

Aveiro, M, C., Garcia, A, P, U., \& Driusso, P. (2009) Efetividade de intervenções fisioterapêuticas para o vaginismo: uma revisão da literatura. Fisioterapia e Pesquisa, 16(3), 279-283.

Baracho, E. (2007) Fisioterapia Aplicada à Obstetrícia, Uroginecologia e Aspectos de Mastologia. (4a ed.), Guanabara Koogan.

Baracho, E. (2012) Fisioterapia Aplicada à saúde da mulher. (5a ed.), Guanabara Koogan.

Delgado, A. M.; Ferreira, I. S. V.; \& Sousa, M. A. (2015) Recursos fisioterapêuticos utilizados no tratamento das disfunções sexuais femininas. Revista Cientifica da Escola da Saúde, 4(1), 47-56.

Etienne, M., Abreu; W., \& Michelle. C. (2006) Disfunções sexuais femininas: a fisioterapia como recurso terapêutico. LPM; $304 p, 2006$.

Goulart, M. G. (2013) Qualidade de vida e satisfação sexual em mulheres com vaginismo antes e após o tratamento fisioterapêutico. 65p, 2013. Trabalho de Conclusão de Curso (graduação em Fisioterapia) - Ciências da Saúde, Universidade do Extremo Sul Catarinense - UNESC, Criciúma, 2013.

Haylen, B., Ridder, D., Freeman, R. M., Swift, S. E., Berghmans, B. Lee, J., Monga, A., Petri, E., Rizk, D., Sand, P., \& Schaer, G. An International Urogynecological Association (IUGA)/International Continence Society (ICS) joint report on the terminology for female pelvic floor dysfunction. Int Urogynecol J. 2010; 21 (5): 26.

Lima, R. G. R.; Silva, S. L. D. S.; Freire, A. D. B.; Barbosa, L. M. A. (2016) Tratamento fisioterapêutico nos transtornos sexuais dolorosos femininos: revisão narrativa. Faculdade Estácio, 1(1), 2-10.

Lucheti, G. C.; Martins, T.; \& Fernandes, I. (2019) Efeito da massagem perineal no tratamento da disfunção sexual dispareunia. Centro Universitário Uniamérica, Foz do Iguaçu/PR, 2019.

Matthes, Â. D. C. S. (2019) Revista Brasileira de Sexualidade Humana. Abordagem atual da dor na relação sexual (dispareunia). Revista Brasileira de Sexualidade Humana, 30(1), 14-22, 2019.

Maldonado, P. A., \& Wai, C. Y. Pelvic Organ Prolapse New Concepts in Pelvic Floor Anatomy. Obstet Gynecol Clin N Am 2016; 43:15-26.

Mannella, P., Palla, G., Bellini, M., \& Simoncini, T. The female pelvic floor through midlife and aging.Maturitas 2013; 76(3):230-4.

Mendonça, C. R. D.; Silva, T. M.; Arrudai, J. T.; Zapata, M. T. A. G.; \& Amaral, W. N. D. (2012) Função sexual feminina: aspectos normais e patológicos, prevalência no brasil, diagnóstico e tratamento, 40(4).

Moltedo-Perfetti, A.; NardI, B.; \& Arimatea, E. (2014) Coherencia sistémica e identitaria en mujeres con vaginismo primario. Rev. chil. obstet. Ginecol. 79(1), 56-63.

Muammar, T.; Mcwalter, P.; Alkhenizan, A.; Shoukri, M.; Gabr, A.; Muammar, AA. (2015) Management of vaginal penetration phobia in Arab women: a retrospective study. Ann Saudi Med. 32(5), 120-126.

Narang, T.; Garima; Singh, S. M. (2016) Psychosexual disorders and dermatologists. Indian Dermatology Online Journal. 7(3), 149, 2016.

Palma, P C; Rodrigues; Berghmans, B; Seleme, M, R. (2014) Urofisioterapia: aplicações clínicas da técnicas fisioterapêuticas nas disfunções miccionais e do assoalho pélvico. In: Urofisioterapia: aplicações clínicas das técnicas fisioterapêuticas nas disfunções miccionais e do assoalho pélvico. 2014

Pereira A. S. et al. (2018). Metodologia da pesquisa científica. UFSM.

Prendergast S, Rummer E, Kotarinos R. Treating vulvodynia with manual physical therapy. Phys Ther. 8(3):7-12

Rao, T. S. S.; Nagaraj, A. K. M. (2015) Female sexuality. Indian Journal of Psychiatry. 57(2), S296-S302.

Silva, D. J. R. D.; \& Abreu, A. H. D. (2014) Recursos fisioterapêuticos para as disfunções sexuais femininas: uma revisão literária. Revista Hórus, 9(1), 53-66.

Tomen, A.; Latorre, G. F. S; Fracaro, G.; \& Nunes, E. F. C. (2015) A fisioterapia pélvica no tratamento de mulheres portadoras de vaginismo. Revista de Ciências Médicas, 121- 130 .

Tomen, A.; Fracaro, G.; Nunes, E., Feio, C.; Latorre, G., \& Fernando, S.. (2016) A fisioterapia pélvica no tratamento de mulheres portadoras de vaginismo. Revista de Ciências Médicas, 24(3), 121-130, 2016 